Rev. Biol. Trop. 52(1): 201-212, 2004

www.ucr.ac.cr www.ots.ac.cr www.ots.duke.edu

\title{
Estructura trófica de la asociación de peces intermareales de la costa rocosa del norte de Chile
}

Viviana Berrios C. \& Mauricio Vargas F.

Departamento de Ciencias del Mar, Universidad Arturo Prat, Casilla 121, Iquique, Chile; viviana.berrios@cec.unap.cl

Recibido 18-VII-2001. Corregido 10-XII-2002. Aceptado 30-I-2003.

\begin{abstract}
The trophic relationships of 13 intertidal fish species collected in northern Chile $\left(20^{\circ} 18^{\prime} \mathrm{S}\right.$ and $20^{\circ} 54$ 'S) from July 1997 to July 1998 , the stomach content analysis of 820 specimens, were investigated. The importance of the prey items was assessed by means of gravimetric and frequency of ocurrence methods, together with the Shannon-Wiener (trophic diversity) and Pianka (trophic overlap) indexes. The results allowed to recognize a fish assemblage composed of: $46 \%$ of carnivorous species, preying mainly on porcelain crabs, polichaetes and minor crustaceans; $23 \%$ of herviborous species, preying mainly on chlorophitic algae; $31 \%$ of omnivorous species, preying mainly on chlorophitic algae, Copepoda and Gastropoda. The carnivorous fishes Cheilodactylus variegatus, Helcogrammoides chilensis, Labrisomus philippii and the omnivorous Oplegnatus insignis showed euriphagic tendencies $(>2.0$ bits), however, the major trophic overlap $(>0.90)$ was observed among herbivorous and omnivorous fishes. Finally, the increase of fish species towards lower latitudes and the increase of herbivory and omnivory levels in the rocky intertidal shore were analyzed.
\end{abstract}

Key words: Trophic structure, intertidal fish assemblage, northern Chile.

El incremento de la riqueza y diversidad de especies desde regiones polares hacia las tropicales, ha sido ampliamente documentado para peces del Pacífico sur oriental (Mann 1954, Arancibia 1992, Sielfeld et al. 1995, Sielfeld y Vargas 1996). Moreno et al. (1979), señala que el incremento de peces submareales de la costa de Chile, puede ser explicado por la estructuración trófica de estas asociaciones, debido al aumento del número de peces herbívoros, particularmente de la familia Kyphosidae hacia el norte del país (Regiones I, II, III y IV) (Jaksic 1997).

Lo anterior se basa en la hipótesis de la heterogeneidad espacial de MacArthur, que señala mayores posibilidades de microhábitat hacia latitudes bajas, otorgadas por un sustrato rocoso más profundo y por un cinturón de macrófitas más extenso, lo que provee una mayor biomasa de presas y áreas de refugio en la zona cubierta por ellas.
Cabe señalar, que de las 11 especies de Kyphosidae registradas en Chile (Pequeño 1989), sólo Girella laevifrons (Tschudi 1844), Medialuna ancietae (Chirichigno 1983) y Graus nigra (Philippi 1887) son comunes en la región norte, presentando hábitos herbívoros (Vial y Ojeda 1990), omnívoros (Vargas et al. 1999) y carnívoros (Fuentes 1982) respectivamente. Por lo tanto, el número de peces herbívoros submareales queda restringido solamente al kifósido G. laevifrons, y a la "jerguilla" Aplodactylus punctatus (Valenciennes 1831) de la familia Aplodactylidae (Núñez y Vásquez 1987, Cáceres et al. 1994), situación similar a lo registrado para la región central de Chile (Moreno et al. 1979). Esto último nos permite evidenciar, que el incremento latitudinal de los peces herbívoros submareales en Chile no es claro, sin embargo puede ser explicado al considerar a los peces intermareales, particularmente los blénidos. 
En Chile, las investigaciones sobre la estructura y organización trófica de las asociaciones ícticas intermareales son en general escasas y referidas principalmente a la región central del país (Stepien 1990, Varas y Ojeda 1990, Muñoz y Ojeda 1997, 1998), a través de las cuales es posible observar que los niveles tróficos de estas asociaciones, se componen de aproximadamente $65 \%$ de carnívoros y $35 \%$ de omnívoros-herbívoros, estando los herbívoros representados exclusivamente por el blénido Scartichthys viridis (Valenciennes 1836) de acuerdo con Muñoz y Ojeda (1997).

El objetivo de este estudio es caracterizar la estructura trófica de la asociación de peces en la zona intermareal rocosa del norte de Chile, como una forma de contribuir al conocimiento del incremento latitudinal de peces herbívoros en estos ambientes, respecto de lo observado para la región central de Chile por Stepien (1990), Varas y Ojeda (1990) y Muñoz y Ojeda (1997).

\section{MATERIALES Y MÉTODOS}

El área de estudio comprendió cinco sectores de pozas intermareales, ubicados entre el campus Huayquique del Departamento de Ciencias del Mar (Universidad Arturo Prat) (2018' S) y Pabellón de Pica (2054' S) (Fig. 1). El período de estudio contempló los meses de julio de 1997 a julio de 1998, durante el cual se realizaron recolecciones mensuales (27 muestreos) en un total de 17 pozas del intermareal rocoso medio-inferior, principalmente en la baja marea, cuyos volúmenes fueron de 2.53 a $35.9 \mathrm{~m}^{3}$ (Berrios y Vargas 2000).

Para la captura de los peces, se utilizó de manera opcional el anestésico BZ- 20 y rotenona, realizando posteriormente la recolección por medio de redes de mano. Los peces capturados fueron fijados directamente en formalina al 10\%, aquellos que presentaron tallas adultas fueron inyectados intraperitonialmente para detener la digestión. Se les guardó luego en frascos plásticos para su análisis posterior en el laboratorio, donde fueron identificados, conta-

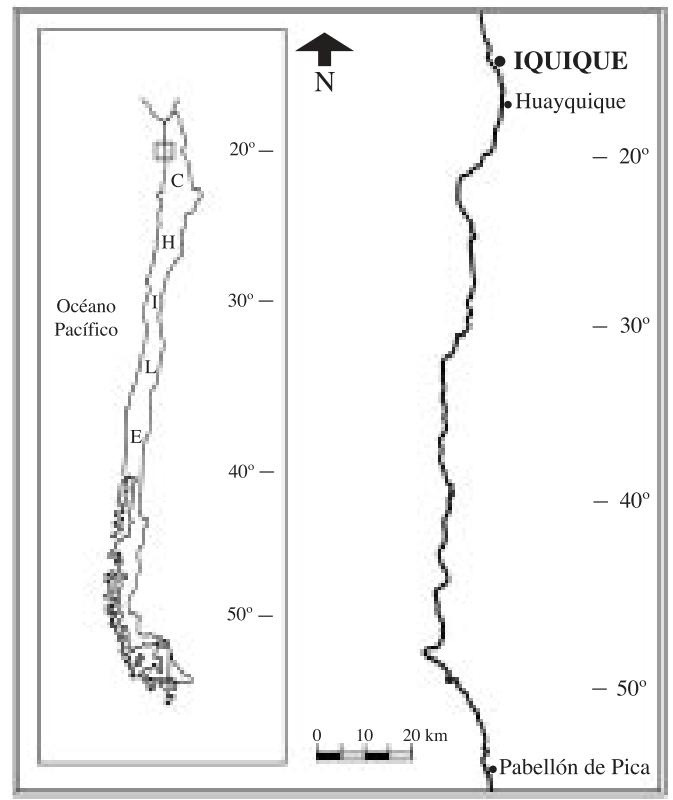

Fig. 1. Ubicación geográfica de los sectores estudiados en el litoral de la Provincia de Iquique.

Fig. 1. Geographical location of the study sites in the shore off Province of Iquique.

dos y medidos, utilizando para ello literatura especializada (i.e. Mann 1954, Chirichigno 1974, Greenfield y Woods 1980, Oyarzún y Pequeño 1989) y un vernier de $0.01 \mathrm{~cm}$ de precisión.

El contenido del tracto digestivo fue vaciado en una cápsula Petri, lavado con agua destilada y secado con papel absorbente, para luego ser observado bajo lupa estereoscópica Nikon (mod. S.M.Z.-10). Los especímenes presas se identificaron sobre la base de claves y literatura especializada, de Retamal (1981) y Guzmán (1999) para crustáceos, Basly (1983) y Guzmán et al. (1998) para moluscos y Boltovskoy (1981) para microinvertebrados. Posteriormente los especímenes identificados fueron pesados (peso húmedo), a través de una balanza analítica de precisión $0.0001 \mathrm{~g}$.

El estudio del espectro trófico de la asociación íctica, consideró un análisis del total de los estómagos de los peces capturados, sin distinción espacial ni temporal, dado que las 
zonas se encuentran relativamente cercanas entre sí. Los contenidos estomacales de los peces fueron reunidos en grupos taxonómicos superiores y analizados por los métodos de Frecuencia de aparición $(\% \mathrm{~F})$ y Gravimétrico (\%G). (Berg 1979, Hyslop 1980).

El primero $(\% \mathrm{~F})$, considera el número de veces en que el espécimen-presa aparece en el total de los estómagos con contenidos, expresándolos en porcentaje.

Con el método gravimétrico $(\% \mathrm{G})$, el peso del espécimen-presa se calcula con respecto al peso total de los especímenes y se expresa en porcentaje.

La condición herbívora de los peces analizados, consideró la presencia del ítem "alga" con $100 \%$ de frecuencia de aparición en los estómagos. Adicionalmente se realizó un reconocimiento cualitativo de la composición específica de las algas, las cuales fueron determinadas por un especialista. Los datos fueron analizados mediante el porcentaje de frecuencia, utilizando para el caso de los peces omnívoros, solo los estómagos que presentaron algas.

Para cada especie íctica, la diversidad trófica se calculó mediante el índice de ShannonWiener (H'), donde pi es la proporción gravimétrica del i-ésimo taxon de presa y $\mathrm{S}$ es el número de táxones de presas. Mediante la comparación de los valores de diversidad, se consideró el carácter eurifágico de los depredadores cuando los valores de H' son altos y el estenofágico cuando los valores de H' son bajos (Berg 1979).

$$
H^{\prime}=\underset{i=11}{-\sum_{i}}\left(\text { pi } x \log _{2} p i\right)
$$

Por último, para determinar las relaciones tróficas entre los peces, se utilizó el índice de superposición (Oxy) de Pianka (1973) cuya formula es: Oxy $=\Sigma\left(\right.$ pxi,pyi) $/ \sqrt{ }\left(\Sigma \mathrm{pxi}^{2}, \Sigma \mathrm{pyi}^{2}\right)$, donde pxi y pyi son los valores del porcentaje gravimétrico que le corresponde a la especiepresa en las dietas del pez uno y pez dos respectivamente. Se considera que existe superposición cuando el índice supera el valor de 0.6 (teniendo presente que este varía entre cero y uno) (Brodeur y Pearcy 1990), lo que indica un consumo de especímenes-presas similares.

Para la determinación de los grupos tróficos, se realizó un análisis de cluster de similitud, a partir del índice de superposición mencionado anteriormente. En la elaboración del dendrograma, se aplicó el método de agrupamiento UPGMA (Unweighted Pair Group Method Average), utilizando para ello las medidas aritméticas. La significancia del agrupamiento en el dendrograma se determinó mediante el método desarrollado por Arancibia (1988).

\section{RESULTADOS}

Se analizó el contenido estomacal de 820 ejemplares (199 estómagos vacíos), pertenecientes a 13 especies (Cuadro 1), de las cuales G. nigra, Auchenionchus microcirrhis (Valenciennes 1836), Gobiesox marmoratus (Jenyns 1842), Cheilodactylus variegatus (Valenciennes 1833), Helcogrammoides chilensis (Cancino 1955) y Labrisomus philippii (Steindachner 1866) fueron registradas como carnívoras; Oplegnathus insignis (Kner 1867), Nexilosus latifrons (Tschudi 1844), Tomicodon chilensis (Brisout de Barneville 1846) y Anisotremus scapularis (Tschudi 1844) como omnívoras; y G. laevifrons, Scartichthys gigas (Steindachner 1876) y $S$. viridis como herbívoras.

Las especies con hábitos carnívoros, presentaron como presas más importantes a crustáceos porcelánidos, peces, poliquetos, insectos y crustáceos menores (i.e. isópodos, copépodos, anfípodos). G. nigra, G. marmoratus, H. chilensis y L. philippii depredaron crustáceos decápodos principalmente porcelánidos (> 16\% biomasa total), y A. microcirrhis y C. variegatus sobre peces $(67 \%)$ y poliquetos (29\%) respectivamente. Las presas más frecuentes para G. nigra y A. microcirrhis fueron crustáceos porcelánidos, mientras que los microcrustáceos lo fueron en G. marmoratus 


\section{CUADRO 1}

Número de estómagos analizados $(N)$, número de estómagos llenos $(N c)$, ámbito de tallas y proporción sexual de las especies de peces capturadas en pozas intermareales en el norte de Chile

TABLE 1

Analysed stomach number $(N)$, full stomach number $(N c)$, size ranks an sex rate of the fish species captured in intertidal pools in northern Chile

\begin{tabular}{|c|c|c|c|c|c|c|c|c|}
\hline \multirow{2}{*}{ Especies } & \multirow[t]{2}{*}{$\mathrm{N}$} & \multirow[t]{2}{*}{$\mathrm{Nc}$} & \multicolumn{3}{|c|}{ Talla } & \multicolumn{3}{|c|}{ Sexo $(\%)$} \\
\hline & & & Ambito & Media & Desv. & Hembra & Macho & Indet. \\
\hline \multicolumn{9}{|l|}{ Orden Gobiesociformes } \\
\hline \multicolumn{9}{|l|}{ Gobiesocidae } \\
\hline Gobiesox marmoratus & 12 & 10 & $3.6-8.0$ & 5.6 & 1.23 & 25.0 & 8.3 & 66.6 \\
\hline Tomicodon chilensis & 14 & 10 & $1.7-4.8$ & 3.24 & 1.07 & 42.8 & - & 57.1 \\
\hline \multicolumn{9}{|l|}{ Orden Perciformes } \\
\hline \multicolumn{9}{|l|}{ Haemulidae } \\
\hline Anisotremus scapularis & 140 & 102 & $2.8-7.9$ & 4.45 & 0.97 & - & - & 100 \\
\hline \multicolumn{9}{|l|}{ Kyphosidae } \\
\hline Girella laevifrons & 140 & 134 & $1.4-12.9$ & 5.21 & 2.08 & - & - & 100 \\
\hline Graus nigra & 38 & 31 & $7.4-15.8$ & 11.02 & 2.26 & - & - & 100 \\
\hline \multicolumn{9}{|l|}{ Oplegnathidae } \\
\hline Oplegnathus insignis & 45 & 17 & $2.9-4.0$ & 3.2 & 0.46 & 一 & 一 & 100 \\
\hline \multicolumn{9}{|l|}{ Pomacentridae } \\
\hline Nexilosus latifrons & 32 & 19 & $1.7-2.9$ & 2.43 & 0.52 & - & 一- & 100 \\
\hline \multicolumn{9}{|l|}{ Cheilodactylidae } \\
\hline Cheilodactylus variegatus & 20 & 16 & $6.1-9.8$ & 7.57 & 0.82 & - & - & 100 \\
\hline \multicolumn{9}{|l|}{ Blennidae } \\
\hline Scartichthys gigas & 100 & 89 & $2.9-15.8$ & 7.38 & 2.97 & 43.1 & 30.5 & 26.3 \\
\hline Scartichthys viridis & 100 & 99 & $3.3-16.5$ & 7.44 & 3.24 & 34.3 & 42.9 & 22.6 \\
\hline \multicolumn{9}{|l|}{ Tripterygiidae } \\
\hline Helcogrammoides chilensis & 11 & 9 & $4.0-5.6$ & 5.01 & 0.52 & 36.3 & 36.3 & 27.2 \\
\hline \multicolumn{9}{|l|}{ Labrisomidae } \\
\hline Auchenionchus microcirrhis & 59 & 44 & $1.01-25.5$ & 9.43 & 6.17 & 14.0 & 5.2 & 80.7 \\
\hline Labrisomus philippii & 109 & 41 & $3.3-9.1$ & 5.95 & 1.65 & - & - & 100 \\
\hline
\end{tabular}

(isópodos 50\%), C. variegatus (copépodos $81 \%$ ), H. chilensis (anfípodos e isópodos $56 \%$ ) y L. philippii (anfípodos 39\%). De las especies carnívoras, L. philippii fue la que presentó una mayor tendencia a la eurifagia $\left(\mathrm{H}^{\prime}=2.7\right)$ (Cuadro 2).

Por otra parte, en los peces omnívoros se registraron como ítemes principales en biomasa (> 35\%), a macroalgas de los grupos ulvales y ectocarpales. Las presas restantes correspon- dieron a invertebrados menores, destacando los copépodos por su alta frecuencia de aparición en la dieta de $N$. latifrons $(74 \%)$ y $O$. insignis (53\%), presentando además, ésta última especie, un amplio espectro alimentario $\left(\mathrm{H}^{\prime}=2.3\right)$ (Cuadro 3).

Las especies herbívoras presentaron una fuerte selección sobre macroalgas, principalmente clorófitas del tipo ulvales y cladoforales, las que corresponden a más del $90 \%$ de la 
CUADRO 2

Porcentaje de frecuencia de aparición $(\% F)$ y gravimétrico $(\% G)$ de los ítemes presas registrados en seis especies de peces de hábitos carnívoros, presentes en la zona intermareal rocosa del norte de Chile

TABLE 2

Frequency of occurrence $(\% F)$ and gravimetric $(\% G)$ percentege of the prey items for six carnivorous fish species, in the rocky intertidal zone in northern Chile

\begin{tabular}{|c|c|c|c|c|c|c|c|c|c|c|c|c|}
\hline \multirow[b]{3}{*}{ Itemes presas } & \multicolumn{12}{|c|}{ Consumidores } \\
\hline & \multicolumn{2}{|c|}{ G. nigra } & \multicolumn{2}{|c|}{ A. microcirrhis } & \multicolumn{2}{|c|}{ G. marmoratus } & \multicolumn{2}{|c|}{ C. variegatus } & \multicolumn{2}{|c|}{ H. chilensis } & \multicolumn{2}{|c|}{ L. philippii } \\
\hline & $\% \mathrm{~F}$ & $\% \mathrm{G}$ & $\% \mathrm{~F}$ & $\% \mathrm{G}$ & $\% \mathrm{~F}$ & $\% \mathrm{G}$ & $\% \mathrm{~F}$ & $\% \mathrm{G}$ & $\% \mathrm{~F}$ & $\% \mathrm{G}$ & $\% \mathrm{~F}$ & $\% \mathrm{G}$ \\
\hline Poliquetos & & & 2.27 & 0.11 & 10.00 & 0.63 & 43.75 & 28.93 & 11.11 & 0.17 & 36.59 & 10.20 \\
\hline Poliplacóforos & 3.23 & 0.94 & & & 10.00 & 0.13 & & & & & & \\
\hline Gastrópodos & 16.13 & 1.75 & 11.36 & 0.62 & 40.00 & 37.27 & & & & & & \\
\hline Bivalvos & & & 2.27 & 0.06 & & & & & & & & \\
\hline Copépodos & & & & & & & 81.25 & 13.95 & & & & \\
\hline Anfípodos & 12.90 & 0.12 & 9.09 & 0.05 & 20.00 & 0.88 & 43.75 & 18.53 & 55.56 & 17.52 & 39.02 & 7.70 \\
\hline Isópodos & 3.23 & 0.33 & & & 50.00 & 6.31 & & & 55.56 & 19.81 & 21.95 & 10.64 \\
\hline Pagúridos & 3.23 & 1.11 & 6.82 & 0.61 & & & & & & & 17.07 & 16.24 \\
\hline Natantios & 9.68 & 17.74 & 18.18 & 1.82 & & & & & 11.11 & 6.70 & 14.63 & 4.03 \\
\hline Porcelánidos & 90.32 & 66.18 & 90.91 & 29.62 & 40.00 & 48.27 & 12.50 & 6.14 & 44.44 & 24.00 & 17.07 & 17.07 \\
\hline Braquiuros & 3.23 & 0.90 & 2.27 & 0.04 & & & & & 11.11 & 2.34 & 4.88 & 0.39 \\
\hline Decápodo indeterminado & & & 4.55 & 0.09 & 20.00 & 6.38 & 18.75 & 8.44 & 22.22 & 14.96 & 21.95 & 30.87 \\
\hline Huevos de crustáceos & & & & & & & & & 11.11 & 14.51 & & \\
\hline Insectos & 3.23 & 0.01 & & & 10.00 & 0.13 & 37.50 & 24.02 & & & 4.88 & 0.02 \\
\hline Peces & 9.68 & 10.92 & 9.09 & 66.96 & & & & & & & 17.07 & 2.57 \\
\hline $\mathrm{H}^{\prime}$ & 1. & 52 & 1. & 14 & & & 2. & 41 & & & 2.7 & 71 \\
\hline
\end{tabular}

biomasa total y un $100 \%$ de frecuencia. Entre estas especies, $S$. gigas presentó la mayor diversidad ( $\left.\mathrm{H}^{\prime}\right)$ de ítemes presas con un valor tan sólo del 0.44 (Cuadro 3).

El análisis cualitativo del contenido algal, permitió registrar un total de 18 especies, destacándose como las más frecuentes en la dieta de los peces con hábitos herbívoros, las clorófitas Enteromorpha prolifera, E. intestinalis y Cladophora sp., la feófita Ectocarpus sp. y la cianófita Lyngbya sp.; el resto de las algas se presentaron bajo 40\%. Así también, los omnívoros registraron una alta frecuencia de las algas clorófitas E. intestinalis, E. prolifera y Ulva sp.; que para el caso particular del pez $T$. chilensis, se agregan además la feófita Ectocarpus sp. y la rodófita Bangia sp. (Cuadro 4).

El análisis de superposición trófica (Pianka 1973) entre los peces estudiados, mostró que el mayor valor $(>0.90)$ se observó entre las especies omnívoras y herbívoras, exceptuando a $O$. insignis (0.59 a 0.71); mientras que entre las especies carnívoras, la mayor superposición (0.75) se observó entre G. nigra y G. marmoratus, seguido de $H$. chilensis y L. philippii (0.69). Entre los peces omnívoros-herbívoros y carnívoros ocurrieron las menores coincidencias alimentarias $(<0.06)$, con la excepción de la especie omnívora $O$. insignis que presentó una coincidencia mayor con las especies carnívoras C. variegatus (0.47), H. chilensis (0.19) y L. philippii (0.17), debido al alto consumo de microcrustáceos y poliquetos (Cuadro 5).

Por otra parte, en el análisis de agrupamientos (Fig. 2) se observó a un nivel de similitud promedio de $62 \%$, la presencia de un grupo principal compuesto por cuatro especies omnívoras (N. latifrons, T. chilensis, A. scapularis y $O$. insignis) y tres herbívoras (G. laevifrons, $S$. viridis y $S$. gigas), las cuales presentaron una fuerte selección por las algas principalmente clorófitas. Los peces de hábitos carnívoros en tanto, mostraron similitudes bajas, debido a la mayor variedad de presas en 


\section{CUADRO 3}

Porcentaje de frecuencia de aparición $(\% F)$ y gravimétrico $(\% G)$ de los ítemes presas registrados en cuatro especies de peces de hábitos omnívoros y tres especies de hábitos herbívoros, presentes en la zona intermareal rocosa del norte de Chile

\section{TABLE 3}

Frequency of occurrence $(\% F)$ and gravimetric $(\% G)$ percentage of the prey items for four omnivorous and three herbivorous fish species, in the rocky intertidal zone in the northern Chile

\begin{tabular}{|c|c|c|c|c|c|c|c|c|c|c|c|c|c|c|}
\hline \multirow[b]{3}{*}{ Itemes presas } & \multicolumn{14}{|c|}{ Consumidores } \\
\hline & \multicolumn{2}{|c|}{ O. insignis } & \multicolumn{2}{|c|}{ N. latifrons } & \multicolumn{2}{|c|}{ A. scapularis } & \multicolumn{2}{|c|}{ T. chilensis } & \multicolumn{2}{|c|}{ S. viridis } & \multicolumn{2}{|c|}{ S. gigas } & \multicolumn{2}{|c|}{ G. laevifrons } \\
\hline & $\% \mathrm{~F}$ & $\% \mathrm{G}$ & $\% \mathrm{~F}$ & $\% \mathrm{G}$ & $\% \mathrm{~F}$ & $\% \mathrm{G}$ & $\% \mathrm{~F}$ & $\% \mathrm{G}$ & $\% \mathrm{~F}$ & $\% \mathrm{G}$ & $\% \mathrm{~F}$ & $\% \mathrm{G}$ & $\% \mathrm{~F}$ & $\% \mathrm{G}$ \\
\hline Macroalgas & 23.53 & 36.80 & 36.84 & 83.86 & 74.51 & 77.23 & 90.00 & 98.53 & 100.00 & 98.06 & 100.00 & 93.55 & 100.00 & 98.83 \\
\hline Poliquetos & 11.76 & 14.67 & & & 1.96 & 0.96 & & & 1.01 & $<0.01$ & 1.12 & 0.01 & 0.75 & $<0.01$ \\
\hline Gastrópodos & & & & & & & 10.00 & 1.47 & 5.05 & 0.62 & 11.24 & 0.58 & 1.49 & 0.05 \\
\hline Bivalvos & & & & & & & & & 4.04 & $<0.01$ & 2.25 & 0.19 & & \\
\hline Copépodos & 52.94 & 23.93 & 73.68 & 16.13 & 30.39 & 0.61 & & & & & & & 2.99 & 0.10 \\
\hline Anfípodos & 11.76 & 14.67 & & & 13.73 & 2.76 & & & 2.02 & $<0.01$ & 2.25 & $<0.01$ & 6.72 & 0.21 \\
\hline Ostrácodos & & & & & 37.25 & 3.35 & & & & & & & & \\
\hline Isópodos & & & & & 2.94 & 0.33 & & & & & 1.12 & $<0.01$ & 0.75 & $<0.01$ \\
\hline Cirripedios & 5.88 & 3.57 & & & & & & & 5.05 & 1.32 & 5.62 & 1.52 & & \\
\hline Natantios & & & & & 0.98 & 1.09 & & & & & & & & \\
\hline Porcelánidos & 11.76 & 6.36 & & & 0.98 & 0.23 & & & 1.01 & $<0.01$ & 5.62 & 4.07 & & \\
\hline Decápodo indeterminado & & & & & 13.73 & 4.35 & & & 1.01 & $<0.01$ & 1.12 & 0.07 & 5.97 & 0.80 \\
\hline Huevos de crustáceos & & & & & 0.98 & 4.40 & & & & & & & 0.75 & $<0.01$ \\
\hline Insectos & & & & & 5.88 & 0.35 & & & 1.01 & $<0.01$ & 1.12 & $<0.01$ & 0.75 & 0.01 \\
\hline Peces & & & & & 1.96 & 4.35 & & & & & & & & \\
\hline $\mathrm{H}^{\prime}$ & 2.2 & & 0.6 & 64 & 1.4 & 44 & 0.1 & 11 & 0. & 5 & 0. & 14 & 0.1 & 10 \\
\hline
\end{tabular}

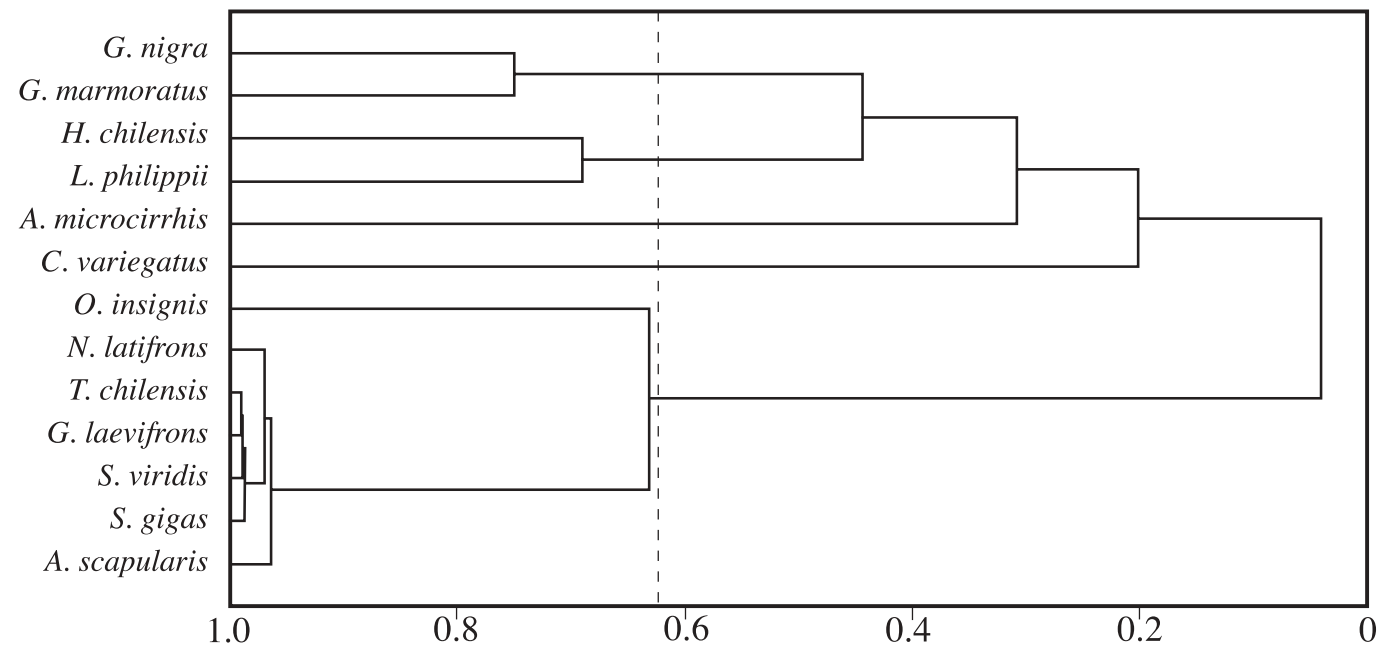

Fig. 2. Similitud trófica de 13 especies de peces presentes en la zona intermareal rocosa del norte de Chile. El nivel medio de similitud corresponde a $62 \%$.

Fig. 2. Tophic similatity of 13 fish species in the rocky intertidal zone in northern Chile. The mean level of similarity corresponds to $62 \%$. 
CUADRO 4

Porcentaje de frecuencia de aparición de las especies de algas registradas en los estómagos (n) de peces omnívoros y herbívoros presentes en pozas intermareales del norte de Chile

TABLE 4

Frequency of occurrence percentage of the algae species registered in the stomach (n) of omnivorous and herbivorous fish species, in intertidal pools in northern Chile

\begin{tabular}{|c|c|c|c|c|c|c|c|}
\hline & & & & Consumidores & & & \\
\hline Itemes algas & $\begin{array}{l}\text { O. insignis } \\
n=4\end{array}$ & $\begin{array}{l}\text { N. latifrons } \\
\mathrm{n}=7\end{array}$ & $\begin{array}{c}\text { A. scapularis } \\
\mathrm{n}=76\end{array}$ & $\begin{array}{l}\text { T. chilensis } \\
\mathrm{n}=9\end{array}$ & $\begin{array}{l}\text { S. viridis } \\
\mathrm{n}=99\end{array}$ & $\begin{array}{c}\text { S. gigas } \\
n=89\end{array}$ & $\begin{array}{l}\text { G. laevifrons } \\
\mathrm{n}=134\end{array}$ \\
\hline Cyanophita & & & & & & & \\
\hline Lyngbya sp. & & & 5.3 & 44.4 & 49.5 & 55.1 & 49.3 \\
\hline Indet. & & & & 22.2 & 35.4 & 21.3 & 26.9 \\
\hline CLOROPHYTA & & & & & & & \\
\hline Ulvales & & & & & & & \\
\hline Enteromorpha prolifera & 100.0 & 71.4 & 23.7 & 77.8 & 57.6 & 47.2 & 70.1 \\
\hline Enteromorpha intestinalis & & 85.7 & 59.2 & 66.7 & 53.5 & 40.4 & 72.4 \\
\hline Ulva sp. & 75.0 & 42.9 & 51.3 & 66.7 & 6.1 & 6.7 & 46.3 \\
\hline Cladoforales & & & & & & & \\
\hline Chaetomorpha sp. & & & 2.6 & & 1.0 & 3.4 & 13.4 \\
\hline Cladophora sp. & & & 22.4 & 55.6 & 46.5 & 55.1 & 53.7 \\
\hline PHAEOPHYTA & & & & & & & \\
\hline Ectocarpales & & & & & & & \\
\hline Bachellotia sp. & & & 2.6 & & 1.0 & & 19.4 \\
\hline Ectocarpus sp. & 25.0 & & 6.6 & 66.7 & 53.5 & 55.1 & 54.5 \\
\hline Inschia sp. & & & & 44.4 & 34.3 & 38.2 & 22.4 \\
\hline Felmania sp. & & & & & 4.0 & 1.1 & 0.7 \\
\hline $\begin{array}{l}\text { RHODOPHYTA. } \\
\text { Bangiales }\end{array}$ & & & & & & & \\
\hline Bangia sp. & & & 1.3 & 66.7 & 27.3 & 28.1 & 23.9 \\
\hline Porphyra sp. & & & & 11.1 & & & 2.2 \\
\hline Gelidiales & & & & & & & \\
\hline Gelidium sp. & & & 11.8 & & 42.4 & 14.6 & 3.7 \\
\hline Pterocladia sp. & & & 2.6 & 44.4 & 38.4 & 43.8 & 12.7 \\
\hline Ceramiales & & & & & & & \\
\hline Ceramiaceae indet. & & & & 11.1 & 13.1 & 10.1 & 5.9 \\
\hline Poliscyphonia sp. & & & 19.7 & 22.2 & 25.3 & 12.4 & 27.6 \\
\hline Indet. & & 28.6 & & & 29.3 & 17.9 & 1.5 \\
\hline Total de especies & 3 & 4 & 12 & 13 & 17 & 16 & 18 \\
\hline
\end{tabular}

sus dietas, sin embargo se pudo observar que mayoritariamente se alimentaron de crustáceos decápodos, principalmente porcelánidos. No obstante, se debe señalar que dentro de los carnívoros se formaron dos conjuntos, uno compuesto por G. nigra y G. marmoratus cuyo ítem principal fueron crustáceos porcelánidos y otro por H. chilensis y L. philippii, en donde además de porcelánidos consumieron otros decápodos.

\section{DISCUSIÓN}

De acuerdo con los resultados, la asociación íctica estudiada está conformada por seis 
CUADRO 5

Superposición trófica (Pianka 1973) entre 13 especies que componen la asociación de peces presentes en la zona intermareal rocosa del norte de Chile

TABLE 5

Trophic overlap (Pianka 1973) between 13 species that compose the fish assemblage of the rocky intertidal zone in northern Chile

\begin{tabular}{|c|c|c|c|c|c|c|c|c|c|c|c|c|}
\hline Especies & G.n. & A.m. & G.m. & C.v. & H.c. & L.p. & o.i. & N.l. & G.l. & T.c. & S.v. & S.g. \\
\hline G. nigra (G.n.) & 0.00 & 0.53 & 0.75 & 0.11 & 0.52 & 0.37 & 0.11 & 0.00 & 0.00 & 0.00 & 0.00 & 0.03 \\
\hline A. microcirrhis (A.m.) & & 0.00 & 0.31 & 0.05 & 0.20 & 0.19 & 0.04 & 0.00 & 0.00 & 0.00 & 0.00 & 0.01 \\
\hline G. marmoratus (G.m.) & & & 0.00 & 0.13 & 0.50 & 0.39 & 0.10 & 0.00 & 0.00 & 0.00 & 0.00 & 0.03 \\
\hline C. variegatus (C.v.) & & & & 0.00 & 0.31 & 0.41 & 0.47 & 0.04 & 0.00 & 0.00 & 0.00 & 0.00 \\
\hline H. chilensis (H.c.) & & & & & 0.00 & 0.69 & 0.19 & 0.00 & 0.00 & 0.00 & 0.00 & 0.01 \\
\hline L. philippii (L.p.) & & & & & & 0.00 & 0.17 & 0.00 & 0.00 & 0.00 & 0.00 & 0.01 \\
\hline O. insignis (O.i.) & & & & & & & 0.00 & 0.71 & 0.59 & 0.59 & 0.60 & 0.62 \\
\hline N. latifrons (N.l.) & & & & & & & & 0.00 & 0.97 & 0.97 & 0.97 & 0.97 \\
\hline G. laevifrons (G.l.) & & & & & & & & & 0.00 & 0.99 & 0.99 & 0.99 \\
\hline T. chilensis (T.c.) & & & & & & & & & & 0.00 & 0.99 & 0.99 \\
\hline S. viridis (S.v.) & & & & & & & & & & & 0.00 & 0.99 \\
\hline S. gigas (S.g.) & & & & & & & & & & & & 0.00 \\
\hline A. scapularis (A.s.) & & & & & & & & & & & & \\
\hline
\end{tabular}

especies carnívoras, cuatro omnívoras y tres herbívoras, correspondiendo las primeras a un $46 \%$, lo cual se asemeja a lo informado en estudios previos sobre la dieta de peces intermareales de Chile central por Stepien (1990), Varas y Ojeda (1990) y Muñoz y Ojeda (1997, 1998), los que indican que más del $50 \%$ de las especies capturadas son carnívoras.

Las especies con hábitos carnívoros, incluyen en su dieta una gran variedad de fauna sésil o de poco movimiento (i.e. cirripedios, poliquetos y gastrópodos) y activa (i.e. decápodos, isópodos, anfípodos, copépodos y peces), de las cuales las presas más importantes son los crustáceos decápodos y microcrustáceos, señalados como presas de altos valores energéticos (Ojeda y Dearborn 1991). Los carnívoros A. microcirrhis y $C$. variegatus, presentan como presas importantes a peces y poliquetos respectivamente, de modo similar a lo registrado para la región central de Chile (Varas y Ojeda 1990, Stepien 1990, Muñoz y Ojeda 1997, 1998), en donde además, agregan en ambas especies a los moluscos gastrópodos como presas importantes. En relación con G. nigra, los resultados discrepan de los registros de Johnson y Fritzsche (1989), quienes le atribuyen a la especie el carácter de omnívora.
En la zona submareal los adultos de las especies mencionadas, si bien mantienen su conducta carnívora, tienen preferencias alimentarias distintas (Fuentes 1982, Vegas y Rojas 1987, Vargas et al. 1999).

Las especies con hábitos omnívoros, $T$. chilensis, A. scapularis, O. insignis y N. latifrons presentaron en su dieta más de un $35 \%$ de biomasa en macroalgas, principalmente clorófitas, mientras que el resto de los ítemes corresponden a pequeños invertebrados. Dentro de las presas más consumidas, los copépodos fueron los más frecuentes en la dieta de $O$. insignis y $N$. latifrons, lo que corrobora la importancia de los microcrustáceos en la alimentación de individuos juveniles, debido a su alto aporte energético. Cabe agregar que en el análisis realizado, se observó que los ejemplares de A. scapularis con tamaño inferior a los $5.0 \mathrm{~cm}$, consumen preferencialmente organismos planctónicos y a medida que la talla aumenta, van incorporando gran cantidad de algas en su dieta, hecho considerado como un cambio trófico durante su ontogenia, antes de su desplazamiento hacia la zona submareal (Morales y Vargas 1998). Por su parte Johnson y Fritzsche (1989), señalan que es común que algunas especies cambien su dieta, siendo más 
frecuente que exista un cambio drástico desde un pez inicialmente carnívoro, a un pez estrictamente herbívoro.

En Chile, estudios sobre la alimentación de estas especies son desconocidos, sin embargo Vegas y Rojas (1986) para la zona intermareal del Perú, infieren que $T$. chilensis presenta hábitos omnívoros debido al bajo número (7-8) de branquiespinas cónicas y cortas y a su dentición, que le permite cortar algas y tomar probablemente pequeños invertebrados del litoral rocoso.

En cuanto a las especies con hábitos herbívoros, G. laevifrons y los blénidos $S$. gigas y $S$. viridis, presentaron una alta dominancia en las pozas intermareales estudiadas (Berrios y Vargas 2000), registrando en su dieta más de $90 \%$ de biomasa algal, principalmente macroalgas clorófitas, feófitas y cianófitas. Además se observó la presencia de pequeños invertebrados, los cuales se asocian a las algas que consumen (Paine y Palmer 1978, Muñoz y Ojeda 1997). Stepien (1990) para la región central del país, registra como especie herbívora a $S$. viridis y G. laevifrons, lo cual concuerda con los presentes resultados, mientras que Muñoz y Ojeda (1997) solamente señalan como herbívora a $S$. viridis, que consume principalmente Gelidium sp. y Ulva sp. Por su parte, Varas y Ojeda (1990) para la misma región, consideran a $G$. laevifrons y $S$. viridis como especies omnívoras, que consumen entre $50 \%$ y $60 \%$ de biomasa de algas del tipo ulvales, correspondiendo las presas restantes a pequeños invertebrados como anfípodos, copépodos, cirripedios y moluscos bivalvos.

Vegas y Rojas (1987) difieren de los resultados referidos a $G$. laevifrons, al señalar que para la zona intermareal del Perú presenta hábitos carnívoros, consumiendo principalmente zooplancton (larvas de crustáceos, pequeños anfípodos gamáridos, pequeños mitílidos, algunos poliquetos y huevos de crustáceos) e indican además que la forma de su estómago y longitud del intestino, confirmarían este tipo de dieta. Al respecto, Fuentes y Cancino (1990) analizan los cambios morfológicos del intestino de esta especie, y señalan que los cambios en las dimensiones del tubo digestivo y en la alimentación, son un mecanismo que permite disminuir los costos de mantenimiento, en la medida en que el balance entre las necesidades y los aportes de energía así lo permitan.

Antecedentes sobre la dieta de $S$. gigas en tanto, son desconocidos, debido a que aparentemente esta especie no es común en la zona intermareal rocosa del centro de Chile, aún cuando Chirichigno (1974) la señala con una distribución desde Guayaquil (Ecuador) a Valdivia (Chile). No obstante, Vegas y Rojas (1987) registran para la zona intermareal del Perú a $S$. gigas, señalándola como omnívora al consumir gran cantidad de algas, restos de erizos y pequeños crustáceos.

Los hábitos alimentarios de las especies analizadas, en general son similares a lo registrado en la región central de Chile, observándose solamente leves cambios en la composición específica de las dietas, lo que no implica necesariamente nichos diferentes. Esta situación ha sido interpretada como cambios producidos en la reacción de escape de las presas y la disponibilidad del alimento en el ambiente (Moreno y Osorio 1977, Moreno et al. 1979). Por su parte Gibson (1982), agrega que la dieta de los individuos puede cambiar marcadamente durante su vida, siendo el tamaño probablemente el factor que determina el tipo de alimentación, más que la edad.

En las zonas templadas de California, los nichos tróficos son ocupados principalmente por peces cotidos, tales como Oligocuttus snyderi y Clinocotus analis (Yoshiyama 1981, Matson et al. 1986), caracterizados por consumir microcrustáceos, esencialmente copépodos. Hacia zonas más tropicales, este grupo es reemplazado por tripterígidos y blénidos, siendo el primero de ellos registrado como microcarnívoro, principalmente consumidor de anfípodos; mientras que el segundo es incluído dentro de los peces con hábitos omnívoros y herbívoros (Kotrschal y Thonson 1986, Stephens et al. 1970). Por lo tanto, el aumento de las especies ícticas de norte a sur en la costa pacífica norte, de acuerdo a lo señalado por 
Thomson y Lehner (1976), tendría relación con la presencia de peces con hábitos omnívoros y herbívoros, situación similar a lo registrado en el presente estudio.

Las relaciones tróficas inter-específicas, mostraron que la asociación íctica intermareal del norte de Chile se estructura por dos gremios tróficos, el primero de ellos compuesto por cuatro especies de hábitos omnívoros y tres herbívoros, las cuales consumen principalmente algas clorófitas (ulvales); el segundo grupo está compuesto por especies de hábitos carnívoros que en su mayoría se alimentan de crustáceos decápodos, principalmente porcelánidos. Esto concuerda con lo encontrado por Muñoz y Ojeda (1997) para la zona intermareal del centro de Chile, donde además registran dentro de los carnívoros un tercer gremio trófico compuesto por especies que consumen microcrustáceos.

La fuerte selección de los peces de hábitos herbívoros-omnívoros sobre pequeñas frondas o rizoides de algas clorófitas (ulvales), las cuales son preferidas por su calidad nutricional (Cáceres et al. 1994), no parece alterar su coexistencia. Al respecto Gibson (1982), señala que dentro de estas asociaciones ícticas existen diferentes patrones de alimentación, como períodos de alimentación, área de alimentación y comportamiento alimentario, los cuales tienden a minimizar la competencia.

En relación con los sectores de alimentación de los peces intermareales, Gibson (1982) señala que estas especies satisfacen sus necesidades alimentarias, realizando excursiones extensivas lejos de las pozas intermareales, principalmente durante la marea alta cuando las condiciones les son más favorables. Este hecho pudo haber ocurrido en el área estudiada, dado que observaciones de terreno permitieron dar cuenta de la ausencia de las algas en las pozas, no obstante estas están presentes en la zona submareal (Soto 1998), por lo cual la presencia de peces herbívoros no se ve afectada.

En conjunto, las especies con hábitos herbívoros y omnívoros registradas en el estudio comprenden $54 \%$ de las especies capturadas (siete especies), lo cual difiere con lo señalado para la zona intermareal del centro de Chile, en donde no sobrepasan 35\% (dos especies). De las especies analizadas, solo G. laevifrons y S. viridis coinciden con lo observado para el centro del país, mientras que el resto no ha sido registrado anteriormente, tales como la especie herbívora $S$. gigas y las omnívoras $T$. chilensis, A. scapularis, $O$. insignis y $N$. latifrons.

La incorporación de especies en el área estudiada, así como también el aumento de las especies herbívoras y omnívoras con relación a la región central de Chile, concuerda con lo indicado por Moreno et al. (1979) para la zona submareal, donde existe un incremento de especies ícticas hacia los trópicos, principalmente de hábitos herbívoros.

\section{AGRADECIMIENTOS}

Los autores expresan sus agradecimientos a Walter Sielfeld K. por impulsar el desarrollo de la presente investigación y por sus valiosos comentarios al manuscrito. Del mismo modo, hacemos extensivos nuestros agradecimientos a F. Patricio Ojeda por facilitar parte del material empleado en terreno, así como también a Raquel Pinto por su colaboración en la identificación de las macroalgas y a cada uno de los estudiantes que participaron en las labores de campo.

\section{RESUMEN}

Se analizaron las interacciones tróficas de 13 especies de peces intermareales, recolectadas en la región norte de Chile (20² 's y 2054'S) durante el período julio 1997 a julio 1998, a partir del contenido estomacal de 820 especímenes. La importancia de los ítemes presa, fue valorada a través de los métodos de frecuencia de aparición y gravimétrico y los índices de Shannon-Wiener (diversidad trófica) y Pianka (sobreposición trófica). Los resultados permitieron reconocer una asociación íctica compuesta por $46 \%$ de especies carnívoras, consumidoras principalmente de crustáceos porcelánidos, poliquetos y crustáceos menores, $23 \%$ de especies herbívoras, consumidoras mayoritariamente de algas clorófitas, feófitas y cianófitas y 31\% de especies omnívoras, consumidoras principalmente de algas clorófitas, copépodos y gastrópodos. Los peces carnívoros Cheilodactylus variegatus, Helcogrammoides chilensis y 
Labrisomus philippii y el omnívoro Oplegnatus insignis registraron una tendencia a la eurifágia $(\mathrm{H}>2.0$ bits $)$, sin embargo la mayor superposicion trófica $(>0.90)$ se observó entre los peces herbívoros y omnívoros. Finalmente se analizó el incremento de especies ícticas hacia latitudes bajas, y el aumento de los niveles de herbivoría y omnivoría en el intermareal rocoso.

\section{REFERENCIAS}

Arancibia, H. 1988. Cluster analysis: the use of a simple statistical method in the identificacion of groups. International Council for the Exploration of the Sea. 18 p.

Arancibia, H. 1992. Distribution patterns of the demersal fish assemblage off central Chile. Biol. Pesq. 21: 4353.

Basly, J. 1983. Moluscos marinos del norte de Chile. Catálogo ilustrativo. Iquique. 49 p.

Berg, J. 1979. Discussion of methods of investigating the food of fishes, with reference to a preliminary study of the prey of Gobiusculus flavescens (Gobiidae). Mar. Biol. 50: 263-273.

Berrios, V. \& M. Vargas. 2000. Estructura del ensamble de peces intermareales de la costa rocosa del norte de Chile. Rev. Biol. Mar. Oceanog. 35: 73-81.

Boltovskoy, D. 1981. Atlas del zooplancton del Atlantico Sudoccidental y metodos de trabajo con el zooplancton marino. Instituto Nacional de Investigación y Desarrollo Pesquero. Mar del Plata, Argentina. 936 p.

Brodeur, R. \& W. Pearcy. 1990. Trophic relations of juvenile Pacific salmon off Oregon and Washington coast. Fish. Bull. U.S. 88: 617-636.

Cáceres, C., L. Fuentes \& F.P. Ojeda. 1994. Optimal feeding strategy of the temperate herbivorous fish Aplodactylus punctatus: the effects of food availability on digestive and reproductive patterns. Oecologia 99: 118-123.

Chirichigno, N. 1974. Clave para identificar los peces marinos del Perú. Instituto del Mar del Perú, Callao. Informe especial 44: 1-387.

Fuentes, H. 1982. Feeding habits of Graus nigra (Labridae) in coastal waters of Iquique, northern Chile. Jap. J. Ichthyol. 29: 95-98.

Fuentes, L. \& J. Cancino. 1990. Cambios morfométricos en el tubo digestivo de juveniles de Girella laevifrons (Kyphosidae) en función de la dieta y del nivel de repleción. Rev. Biol. Mar., Valparaíso 25: 19-26.
Gibson, R. 1982. Recent studies on the biology of intertidal fishes. Oceangr. Mar. Biol. Annu. Rev. 20: 363414.

Greenfield, D. \& L. Woods. 1980. Review of the deep-bodied species of Chromis (Pisces: Pomacentridae) from the eastern pacific, with descriptions of three new species. Copeia 1980 (4): 626-641.

Guzmán, G. 1999. Clave para los crustáceos decápodos anomuros marinos de Chile. Campus. Universidad Arturo Prat. Iquique. 53 p.

Guzmán, N., S. Saá \& L. Ortlieb. 1998. Catálogo descriptivo de los moluscos litorales (Gastropoda y Pelecypoda) de la zona de Antofagasta, $23^{\circ} \mathrm{S}$ (Chile). Estud. Oceanol. 17: 17-86.

Hyslop, E. 1980. Stomach contents analysis. A review of methods an their application. J. Fish. Biol. 17: 411-429.

Jaksic, F. 1997. Ecología de los vertebrados de Chile. Universidad Católica de Chile. Santiago. 262 p.

Johnson, G. \& R. Fritzsche. 1989. Graus nigra, an omnivorous Girellid, with a comparative osteology and comments on relationships of the Girellidae (Pisces: Perciformes). Proc. Acad. Nat. Sci. Philad. 141:1-27.

Kotrschal, K. \& D. Thompson. 1986. Feeding patterns in eastern tropical Pacific blennioid fishes (Teleostei: Tripterygiidae, Labrisomidae, Chaenopsidae, Blenniidae). Oecologia 70: 367-378.

Mann, G. 1954. La vida de los peces en aguas chilenas. Instituto de Investigaciones Veterinarias y Universidad de Chile. Santiago. 342 p.

Matson, R., C. Crabtree \& T. Haglund. 1986. Ichthyofaunal composition and recolonization in a central California tidepool. Calif. Fish and Game 72: 227-231.

Morales, M. \& M. Vargas. 1998. Cambios ontogenéticos en la dieta de Anisotremus scapularis (Perciformes, Haemulidae) del norte de Chile ( $\left.20^{\circ} 11^{\prime} \mathrm{S}-21^{\circ} 19^{\prime} \mathrm{S}\right)$. XVIII Congreso de Ciencias del Mar. Iquique, Chile. 223 p.

Moreno, C. \& C. Osorio. 1977. Bathymetric food habits changes in the Antarctic fish, Notothenia gibberifrons Lönnberg (Pisces: Nototheniidae). Hydrobiologia 55: 139-144.

Moreno, C., W. Duarte \& J. Zamorano. 1979. Variación latitudinal del número de especies de peces en el sublitoral rocoso: una explicación ecológica. Arch. Biol. Med. Exper. 12: 169-178.

Muñoz, A. \& F.P. Ojeda. 1997. Feeding guild structure of a rocky intertidal fish assemblage in central Chile. Env. Biol. Fish. 49: 471-479. 
Muñoz, A. \& F.P. Ojeda. 1998. Guild structure of carnivorous intertidal fishes of the Chilean coast: implications of ontogenetic dietary shifts. Oecologia 114: 563-573.

Nuñez, L. \& J. Vásquez. 1987. Observaciones tróficas y de distribución espacial de peces asociados a un bosque submareal de Lessonia trabeculata. Estud. Oceanol. 6: $79-85$.

Ojeda, F.P. \& J. Dearborn. 1991. Feeding ecology of benthic mobile predators: experimental analyses of their influence in rocky subtidal communities of the Gulf of Maine. J. Exp. Mar. Biol. Ecol. 149: 13-44.

Oyarzún, F. \& G. Pequeño. 1989. Sinopsis de Blennidae de Chile (Osteichthyes, Perciformes). Gayana Zool. 53: $3-40$.

Paine, R. \& R. Palmer. 1978. Syciases sanguineus: a unique trophic generalist from the Chilean intertidal zone. Copeia 1978: 75-81.

Pequeño, G. 1989. Peces de Chile. Lista sistemática revisada y comentada. Rev. Biol. Mar., Valparaíso 24: 1132.

Pianka, E. 1973. The structure of lizard communities. Annu. Rev. Ecol. Syst. 4: 53-74.

Retamal, M. 1981. Catálogo ilustrativo de los crustáceos decápodos de Chile. Gayana Zool. 44: 1-110.

Sielfeld, W., M. Vargas \& R. Fuenzalida. 1995. Peces mesopelágicos frente a la costa norte de Chile (18 $25^{\prime}$ $21^{\circ} 47^{\prime}$ S). Invest. Mar., Valparaíso 23: 83-97.

Sielfeld, W. \& M. Vargas. 1996. Composición y estructura de la ictiofauna demersal en la zona norte de Chile. Invest. Mar., Valparaíso 24: 3-17.

Soto, R. 1998. Estructura comunitaria de macroinvertebrados asociados al disco adhesivo de Lessonia nigres- cens (Bory 1825) del intermareal rocoso en el extremo norte de Chile y relación con el evento El Niño 1991-1992. Tesis de Magister. Universidad de Concepción. Concepción, Chile. 79 p.

Stephens, J. Jr., R. Johnson, G. Key \& J. McCooker. 1970. The comparative ecology of three sympatric species of California blennies of the genus Hypsoblennius Gill (Teleostomi, Blenniidae). Ecol. Monogr. 40: 213-233.

Stepien, C. 1990. Population structure, diets and biogeographic relationships of a rocky intertidal fish assemblage in central Chile: high levels of herbivory in a temperate system. Bull. Mar. Sci. 47: 598-612.

Thomson, D. \& C. Lehner. 1976. Resilience of a rocky intertidal fish community in a physically unstable environment. J. Exp. Mar. Biol. Ecol. 22: 1-29.

Varas, E. \& F.P. Ojeda. 1990. Intertidal fish assemblages of the central chilean coast: diversity, abundance and trophic patterns. Rev. Biol. Mar. 25: 59-70

Vargas, M., R. Soto \& G. Guzmán. 1999. Cambios interanuales en la alimentación de peces submareales del norte de Chile entre los $20^{\circ} 11^{\prime}$ y $20^{\circ} 20^{\prime}$ S. Rev. Biol. Mar. Oceanog. 34: 197-210.

Vegas, M. \& P. Rojas 1987. Estudio de algunos peces marinos relacionados con el litoral rocoso del Perú. Segundo Congreso Latinoamericano sobre Ciencias del Mar. Carácas, Venezuela. pp. 249-263.

Vial, C. \& F.P. Ojeda. 1990. Cephalic anatomy of the herbivorous fish Girella laevifrons (Osteichthyes: Kyphosidae): mechanical considerations of its trophic function. Rev. Chil. Hist. Nat. 63: 247-260.

Yoshiyama, R. 1981. Distribution and abundance patterns of rocky intertidal fishes in central California. Environ. Biol. Fish. 6: 315-332. 